\title{
Which Percutaneous Tracheostomy Method Is Better? A Systematic Review
}

\author{
Alvaro Sanabria MD MSc PhD
}

\begin{abstract}
BACKGROUND: The aim of this study was to assess the different methods of percutaneous tracheostomy in terms of successful performance of the tracheostomy as well as safety. Tracheostomy is the most common procedure performed on the airway for patients in ICUs. Lately, several methods of percutaneous tracheostomy (multiple dilator, progressive dilator, forceps dilation, screwlike dilation, balloon dilation, and translaryngeal) have been described, with theoretical advantages, but there is no consensus about which is better. METHODS: A systematic review with critical appraisal of the literature was done. Literature in multiple databases was searched. Randomized controlled trials comparing different tracheostomy methods were selected. Clinical and methodological characteristics were assessed. A meta-analysis using fixed effect models was planned for statistically homogeneous outcomes. RESULTS: Fourteen randomized controlled trials were included, most of them with small sample sizes and with comparisons of multiple methods. Blue Rhino methods were less difficult for surgeons (risk difference of $14.7 \%$ [95\% CI 8-21.5]) and had more minor bleeding events (risk difference of $-6.3 \%$ [95\% CI -13.58 to 0.8$]$ ). There were no differences in major bleeding events. Statistically, heterogeneity and lack of data impede comparison with other outcomes. CONCLUSIONS: The Blue Rhino method is less difficult and has more minor bleeding events, but physicians also have more experience with this technique. However, trials are underpowered to define the best method. Key words: tracheostomy; meta-analysis; intensive care; surgery; minimally invasive surgical procedures. [Respir Care 2014;59(11):1660-1670. () 2014 Daedalus Enterprises]
\end{abstract}

\section{Introduction}

Tracheostomy is the most common procedure performed on the airway in patients in ICUs. The first attempt was registered on Egyptian papyrus in 3,100 BC; the procedure evolved in the centuries that followed to open tracheostomy. ${ }^{1}$ The open tracheostomy procedure was standard-

\footnotetext{
Dr Sanabria is affiliated with the Head and Neck Service, Hospital Pablo Tobon Uribe, and the Department of Surgery, School of Medicine, Universidad de Antioquia, Medellín, Colombia.

Dr Sanabria was supported by the Universidad de Antioquia and Hospital Pablo Tobon Uribe.

Dr Sanabria has disclosed no conflicts of interest.

Correspondence: Alvaro Sanabria MD MSc PhD, Oncology Unit, Hospital Pablo Tobon Uribe, Calle 78B No. 69-240, Medellín, Colombia. E-mail: alvarosanabria@gmail.com.
}

DOI: $10.4187 /$ respcare. 03050 ized by Jackson in $1909^{1}$ and underwent minor changes during the 20th century. In 1985, Ciaglia et $\mathrm{al}^{2}$ described a modification of open tracheostomy and introduced the Seldinger principle for the antegrade percutaneous approach. The first percutaneous tracheostomy system employed multiple, sequentially larger dilators. However, in 1999, Ciaglia modified his original procedure to the single progressive dilator method known today as Blue Rhino. ${ }^{3}$ Other methods of antegrade percutaneous tracheostomy were reported by Griggs et $\mathrm{al}^{4}$ in 1990 using a one-step dilation of the tracheostoma with rounded-tip forceps, by Frova and Quintel ${ }^{5}$ in 2002 using stomal dilation with a unique screw-like dilating device (PercuTwist), and by Zgoda and Berger ${ }^{6}$ in 2005 and Cianchi et al ${ }^{7}$ in 2010 using a modification of the Blue Rhino device that employed balloon dilation. A retrograde tracheostomy technique called the translaryngeal method was described by Fantoni and Ripamonti ${ }^{8}$ in 1997.

All methods of percutaneous tracheostomy use a modification of the Seldinger technique, which involves punc- 
turing the trachea percutaneously with a sharp hollow trocar through which a guidewire is advanced. The trocar is then withdrawn, and dilators are serially passed over the guidewire, which directs the tip of the dilators into the tracheal lumen. There are some variations in the method of performing and dilating the tracheal stoma; some have theoretical advantages, including providing direct vision when performing the dilation, simplicity, and safety. Nonetheless, there is disagreement about which of these methods is the best in terms of effectiveness and safety, and the choice is often determined by subjective factors, experience, economic issues, and availability. To date, there is only one systematic review that addresses this question. In 2012, Cabrini et al ${ }^{9}$ analyzed 13 randomized controlled trials (RCTs) and suggested that the Blue Rhino method was safer and more effective, and suggested that the translaryngeal method be discarded because of its high number of complications. However, the meta-analysis involved highly heterogeneous evidence and made the assumption that the studies supported equivalence between the methods.

Traditionally, percutaneous tracheostomy has been a subject of interest to both intensive care and anesthesia specialists, but in recent years, it has been used by general surgeons, head and neck surgeons, and otolaryngologists. The subject becomes more critical when percutaneous tracheostomy is used with more complicated patients and when complications need to be managed by these specialists. The aim of this study was to assess the different methods of percutaneous tracheostomy used for ICU patients in terms of successful performance of the tracheostomy, as well as safety relating to outcomes such as bleeding, tracheal tears, false tracts, and performance difficulty.

\section{Methods}

A search was conducted on the MEDLINE, LILACS, Embase, and Cochrane Collaboration databases for RCTs published between January 1985 and November 2013 using the terms tracheostomy, tracheotomy, clinical trial, and percutaneous. An expanded search of each relevant article was performed using Boolean operators. References were explored to identify other articles. Only studies published in the English language were included (Fig. 1).

Following a preliminary search, I reviewed all abstracts; those that dealt with percutaneous tracheostomy methods were selected for further analysis. Inclusion criteria for studies were RCTs of adult ICU subjects who underwent elective tracheostomy. Exclusion criteria were contraindications to percutaneous tracheostomy such as local infection, bleeding disorders, malignant cervical tumors, and spinal trauma. The Ciaglia multiple dilator, Ciaglia Blue Rhino, Ciaglia balloon dilator, Griggs, Fantoni, and PercuTwist methods were analyzed. The main outcome measures were complications and difficulty/impossibility

\section{QUICK LOOK}

\section{Current knowledge}

Tracheostomy is the most common surgical procedure performed in patients in the ICU. Percutaneous tracheostomy at the bedside has widely replaced intra-operative tracheostomy owing to reduced costs and convenience. The optimum technical procedure for percutaneous tracheostomy has not been determined.

\section{What this paper contributes to our knowledge}

A meta-analysis of 14 studies with small patient populations failed to show distinct advantages of any one technique. The multiple dilator method was less difficult but had a slight increase in minor bleeding complications. The composite literature does not allow definitive conclusions on the best percutaneous technique for tracheostomy.

to perform the procedure. Other outcomes such as hypoxemia or hypotension during the procedure reported by each study were recorded but were not considered in the final analysis. Complications were classified into major (bleeding requiring any intervention, defined as major by the original authors, or posterior tracheal tears) and minor (limited bleeding). Outcome follow-up had to be until death or the ICU discharge date. Difficulty was classified subjectively by most authors; for the purpose of this study, a negative outcome was classified as a highly difficult or impossible tracheostomy procedure and having to change the method of tracheostomy.

Data were collected regarding sample size, subject characteristics, surgical procedures, and outcomes. The methodological quality of each study was assessed in accordance with Cochrane Collaboration guidelines for RCTs (random sequence generation, allocation concealment, blinding of participants and personnel, blinding of outcome assessment, incomplete outcome data, selective reporting, and other bias) giving a qualification of risk of bias. Methodological and clinical weaknesses are discussed.

Data from each study were extracted for calculation of risk differences using a $2 \times 2$ table. Statistical analysis was performed with Stata 9.0 software (StataCorp, College Station, Texas), and risk difference $(95 \% \mathrm{CI}$ ) was calculated for each outcome using the Mantel-Haenszel fixed effects model when heterogeneity allowed (Higgins $\left.\mathrm{I}^{2}<50 \%\right)$. Sensitivity analysis adjusting for quality and subgroup analysis by type of method (antegrade vs retrograde) and dilation technique were also planned. Heterogeneity was assessed with the $\mathrm{Q}$ test (significant if $P<.05$ ), and the influence of heterogeneity on the odds ratio was determined with the $\mathrm{I}^{2}$ test. If clinical and methodological 


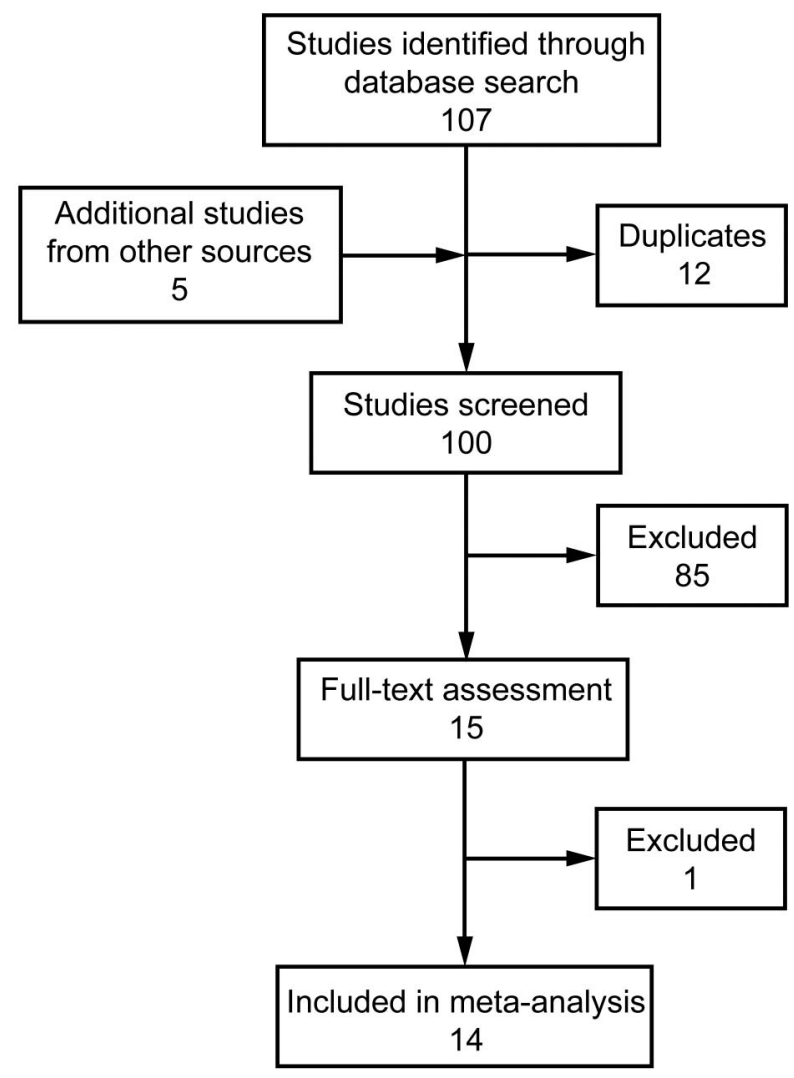

Fig. 1. Flow diagram.

heterogeneities were not found, a meta-analysis of the results was attempted. In other cases, heterogeneity was explained. The results for the intervention effect are presented in a forest plot graph. Recommendations are classified in accordance with levels of evidence.

\section{Results}

A literature search found 100 potential studies; 14 RCTs directly compared 2 methods of percutaneous tracheostomy. The study by Stocchetti et al ${ }^{15}$ was not included because of a lack of information relating to outcome. Study characteristics, events, and quality of evaluation of each selected study are shown in Tables $1-3$.

\section{Ciaglia Multiple Dilator Versus Ciaglia Blue Rhino}

In 2000, Byhahn et $\mathrm{al}^{22}$ compared these 2 methods in 50 subjects, with the multiple dilator method as the control group. There was no mention about operators' experience. Most subjects had cardiovascular or abdominal diseases $(80 \%)$, and the main indication for tracheostomy was sepsis (36\%). Duration of intubation was $\sim 7 \mathrm{~d}$. The length of the procedure was shorter for the Blue Rhino method (165 vs $386 \mathrm{~s}$ ), and the cannulation time was $\sim 17 \mathrm{~d}$. There were no statistically significant differences between oxygenation measurements before or after tracheostomy, and there were no deaths related to the tracheostomy procedure. In 2001, Johnson et $\mathrm{al}^{21}$ also compared these methods in 50 subjects. Operators were trained surgeons or intensive care residents supervised by surgeons. Most subjects were trauma victims (72\%). There were no data about indication for tracheostomy, time of intubation, or tracheostomy. The length of the procedure was shorter for the Blue Rhino method (6. 01 vs $10.01 \mathrm{~min}$ ). There were no deaths related to the tracheostomy procedure.

The risk difference for tracheal ring fracture was $-28 \%$ (95\% CI -49.6 to -6.4 ), the only statistically significant comparison. There were no episodes of major bleeding.

\section{Ciaglia Blue Rhino Versus Ciaglia Balloon Dilator (Blue Dolphin)}

In 2010, Cianchi et $\mathrm{al}^{7}$ compared the Ciaglia Blue Rhino system versus the new Ciaglia balloon dilation system (Blue Dolphin) in 70 subjects, using the Blue Rhino as the control group. Operators were intensivists with experience with the Blue Rhino method. There were no data about the underlying diseases of the subjects, indications, or tracheostomy. Duration of intubation was $\sim 9 \mathrm{~d}$. The operating time in the Blue Rhino group was shorter than that in the Blue Dolphin group (1.5 vs $4 \mathrm{~min}$ ). There were no deaths related to the tracheostomy procedure. The risk difference for minor bleeding was $-34.3 \%$ ( $95 \% \mathrm{CI}-56.3$ to -12.3$)$, and for difficulty was $-22.9 \%$ (95\% CI -39.7 to -6.0 ), which is higher in all respects for the Blue Dolphin method. Comparisons of other outcomes were not statistically significant.

\section{Ciaglia Blue Rhino Versus Griggs Dilating Forceps}

In 2002, Ambesh et al ${ }^{11}$ compared the Ciaglia Blue Rhino versus Griggs dilating forceps methods in 60 subjects, with the Blue Rhino as the control group. Operators were intensivists with previous experience. There were no data about the diseases of the subjects or indications for tracheostomy. Duration of intubation was $\sim 8 \mathrm{~d}$. The operating time was shorter in the Griggs group (6.5 vs $7.5 \mathrm{~min}$ ), but the difference was not statistically significant. Time to decannulation was $\sim 11 \mathrm{~d}$. The increase in peak airway pressure was higher in the Blue Rhino group (16.5 vs $6 \mathrm{~mm} \mathrm{Hg}$ ); this was statistically significant. There were more cases of surgical emphysema in the Griggs group (3 vs 0). In 2004, Añon et al ${ }^{12}$ reported a study of 53 subjects. Operators were intensivists with previous experience. Most subjects had cardiopulmonary diseases $(60 \%)$. Intubation duration was $\sim 17 \mathrm{~d}$. The procedure time was shorter in the Griggs group (7 vs $9 \mathrm{~min}$ ). There were no data about indications for tracheostomy and time to decannulation. 


\section{Percutaneous Tracheostomy Methods}

Table 1. Main Characteristics of Included Studies

\begin{tabular}{|c|c|c|c|c|}
\hline Reference & Subjects & Intervention & Control & Outcome \\
\hline $\begin{array}{l}\text { Cianchi } \\
\text { et }^{7}{ }^{7}\end{array}$ & $\begin{array}{l}\text { Adult subjects in an ICU needing } \\
\text { elective tracheostomy; } \\
\text { excluding infection, injury, or } \\
\text { malignancies on the anterior } \\
\text { part of the neck, brain } \\
\text { hypoperfusion, thyroid gland } \\
\text { hypertrophy, and coagulation } \\
\text { disorder }\end{array}$ & $\begin{array}{l}\text { Ciaglia balloon dilation under } \\
\text { general intravenous } \\
\text { anesthesia with fiberoptic } \\
\text { guidance }\end{array}$ & $\begin{array}{l}\text { Ciaglia Blue Rhino dilation under } \\
\text { general intravenous anesthesia } \\
\text { with fiberoptic guidance }\end{array}$ & $\begin{array}{l}\text { Complications occurring during the } \\
\text { surgical procedure and seen } \\
\text { during the subject's stay in an } \\
\text { ICU such as bleeding, false } \\
\text { passage, inability to complete the } \\
\text { procedure, tracheal tears, } \\
\text { pneumothorax, and mortality }\end{array}$ \\
\hline $\begin{array}{l}\text { Byhahn } \\
\text { et al }{ }^{10}\end{array}$ & $\begin{array}{l}\text { Critically ill adult subjects } \\
\text { requiring elective tracheostomy } \\
\text { because of long-term } \\
\text { ventilation; excluding local } \\
\text { infection, endotracheal } \\
\text { intubation difficulty, distorted } \\
\text { anatomy, and unstable cervical } \\
\text { spine }\end{array}$ & $\begin{array}{l}\text { PercuTwist tracheostomy at } \\
\text { bedside under general } \\
\text { intravenous anesthesia with } \\
\text { fiberoptic guidance }\end{array}$ & $\begin{array}{l}\text { Blue Rhino tracheostomy under } \\
\text { general intravenous anesthesia } \\
\text { with fiberoptic guidance at the } \\
\text { bedside }\end{array}$ & $\begin{array}{l}\text { Complications classified as serious, } \\
\text { intermediate; and minor; } \\
\text { difficulty in dilation and } \\
\text { insertion classified as I (without } \\
\text { difficulty), II (minor difficulties), } \\
\text { III (very difficult but possible), } \\
\text { and IV (impossible, switch to } \\
\text { another tracheostomy method) }\end{array}$ \\
\hline $\begin{array}{l}\text { Ambesh } \\
\text { et al }{ }^{11}\end{array}$ & $\begin{array}{l}\text { Critically ill subjects requiring } \\
\text { tracheostomy because of } \\
\text { prolonged ventilatory support, } \\
\text { airway protection, pulmonary } \\
\text { toilette, or facilitation of } \\
\text { weaning from a ventilator in an } \\
\text { ICU }\end{array}$ & $\begin{array}{l}\text { Griggs tracheostomy under } \\
\text { general intravenous } \\
\text { anesthesia }\end{array}$ & $\begin{array}{l}\text { Blue Rhino tracheostomy under } \\
\text { general intravenous anesthesia }\end{array}$ & $\begin{array}{l}\text { Complications such as bleeding, } \\
\text { infection, decannulation, and } \\
\text { difficulty of insertion; subjective } \\
\text { assessment of dilation and } \\
\text { cannulation; bronchoscopy } \\
\text { assessment of mucosal tears and } \\
\text { circumferential disruption of } \\
\text { tracheal rings }\end{array}$ \\
\hline $\begin{array}{l}\text { Añon } \\
\text { et al }{ }^{12}\end{array}$ & $\begin{array}{l}\text { Critically ill subjects requiring } \\
\text { endotracheal intubation for } \\
\text { mechanical ventilation for } \\
>15 \mathrm{~d} \text {; excluding } \mathrm{F}_{\mathrm{IO}_{2}}>0.8 \\
\text { or PEEP }>10 \mathrm{~cm} \mathrm{H}_{2} \mathrm{O}, \\
\text { cervical spine lesion, } \\
\text { uncorrectable coagulopathy, } \\
\text { anatomical distortion of the }\end{array}$ & $\begin{array}{l}\text { Griggs tracheostomy under } \\
\text { general intravenous } \\
\text { anesthesia at the bedside }\end{array}$ & $\begin{array}{l}\text { Blue Rhino tracheostomy under } \\
\text { general intravenous anesthesia } \\
\text { at the bedside }\end{array}$ & $\begin{array}{l}\text { Complications occurring during the } \\
\text { surgical procedure and seen } \\
\text { during the subject's stay in an } \\
\text { ICU such as bleeding, false } \\
\text { passage, inability to complete the } \\
\text { procedure, tracheal tears, } \\
\text { pneumothorax, and mortality }\end{array}$ \\
\hline
\end{tabular}

Griggs tracheostomy under general intravenous anesthesia with fiberoptic guidance at the bedside

$\begin{array}{cc}\begin{array}{c}\text { Kumar } \\ \text { et al }^{13}\end{array} & \begin{array}{c}\text { Adult subjects requiring } \\ \text { endotracheal intubation; }\end{array}\end{array}$ endotracheal intubation;
excluding high oxygen requirements, high PEEP cervical injuries, coagulopathy, tracheal distortion, previous neck surgery, goiter, obesity, infection, and emergency procedures

Karvandian Adult subjects requiring et $\mathrm{al}^{14} \quad$ endotracheal intubation for lung or neurological disease intubated for 7-10 d; excluding coagulopathy, tracheal distortion, short neck, goiter, obesity, and infection

\section{Van Heurn Critically ill adult subjects} et al ${ }^{16} \quad$ requiring elective tracheostomy because of prolonged intubation, weaning from mechanical ventilation, bronchial toilet, or upper airway obstruction; excluding $\mathrm{PEEP}>10 \mathrm{~mm} \mathrm{Hg}, \mathrm{F}_{\mathrm{IO}_{2}}>0.8$, coagulopathy, local infection, and cervical spine fracture

Cantais Adult subjects in an ICU and et $\mathrm{al}^{17} \quad$ expected to require mechanical ventilation for $>10 \mathrm{~d}$ or prolonged protection of the airway; excluding adverse anatomy, short neck, cervical spine stiffness, coagulation disorder, and high intracranial pressure

Blue Rhino tracheostomy under general intravenous anesthesia with fiberoptic guidance at the bedside
Complications such as difficulty, bleeding, ring fracture, posterior tracheal wall perforation, pneumothorax, and desaturation
Griggs tracheostomy under general intravenous anesthesia with fiberoptic guidance at the bedside

Griggs tracheostomy without fiberoptic guidance

Fantoni tracheostomy under general intravenous anesthesia at the bedside
Blue Rhino tracheostomy under general intravenous anesthesia with fiberoptic guidance at the bedside

Ciaglia multiple dilator tracheostomy without fiberoptic guidance

Complications such as emphysema, hypotension, arrhythmia, bleeding, ring fracture, posterior tracheal wall perforation, pneumothorax, and desaturation

Complications such as hemorrhage false passage and infection; subjective assessment of dilation and cannulation

Griggs tracheostomy under general intravenous anesthesia at the bedside
Complications occurring during the surgical procedure and seen during the subject's stay in an ICU such as bleeding, false passage, inability to complete the procedure, tracheal tears, pneumothorax, and mortality 
Table 1. Continued

\begin{tabular}{|c|c|c|c|c|}
\hline Reference & Subjects & Intervention & Control & Outcome \\
\hline $\begin{array}{l}\text { Nates } \\
\quad \text { et } \mathrm{al}^{18}\end{array}$ & $\begin{array}{l}\text { Adult subjects in an ICU with } \\
\text { upper airway obstruction, } \\
\text { prolonged protection of the } \\
\text { airway, or facilitation of } \\
\text { weaning; excluding infection, } \\
\text { coagulation disorder, adverse } \\
\text { anatomy, previous neck } \\
\text { surgery, pregnancy, burns, and } \\
\text { post-sternotomy }\end{array}$ & $\begin{array}{l}\text { Griggs tracheostomy under } \\
\text { general intravenous } \\
\text { anesthesia at the bedside }\end{array}$ & $\begin{array}{l}\text { Ciaglia multiple dilator } \\
\text { tracheostomy under general } \\
\text { intravenous anesthesia at the } \\
\text { bedside }\end{array}$ & $\begin{array}{l}\text { Complications classified as serious, } \\
\text { intermediate, and minor; } \\
\text { difficulty in dilation and } \\
\text { insertion classified as I (without } \\
\text { difficulty), II (minor difficulties) } \\
\text { III (very difficult but possible) } \\
\text { and IV (impossible, switch to } \\
\text { another tracheostomy method) }\end{array}$ \\
\hline $\begin{array}{l}\text { Montcriol } \\
\text { et al }{ }^{19}\end{array}$ & $\begin{array}{l}\text { Adult subjects in an ICU needing } \\
\text { elective tracheostomy; } \\
\text { excluding adverse anatomy, } \\
\text { short neck, cervical spine } \\
\text { stiffness or trauma, coagulation } \\
\text { disorder, and brain } \\
\text { hypoperfusion }\end{array}$ & $\begin{array}{l}\text { PercuTwist tracheostomy } \\
\text { under general intravenous } \\
\text { anesthesia with fiberoptic } \\
\text { guidance at the bedside }\end{array}$ & $\begin{array}{l}\text { Griggs tracheostomy under } \\
\text { general intravenous anesthesia } \\
\text { with fiberoptic guidance }\end{array}$ & $\begin{array}{l}\text { Complications classified as major } \\
\text { and minor; difficulty in dilation } \\
\text { and insertion }\end{array}$ \\
\hline $\begin{array}{l}\text { Kaiser } \\
\quad \text { et } \mathrm{al}^{20}\end{array}$ & $\begin{array}{l}\text { Adult subjects in an ICU needing } \\
\text { elective tracheostomy because } \\
\text { of expected ventilator } \\
\text { dependence for }>10 \mathrm{~d} \text {, } \\
\text { respiratory insufficiency, and } \\
\text { neurological problems; } \\
\text { excluding adverse anatomy, } \\
\text { short neck, cervical spine } \\
\text { stiffness or trauma, and } \\
\text { coagulation disorder }\end{array}$ & $\begin{array}{l}\text { Griggs tracheostomy under } \\
\text { general intravenous } \\
\text { anesthesia with fiberoptic } \\
\text { guidance at the bedside }\end{array}$ & $\begin{array}{l}\text { Ciaglia multiple dilator } \\
\text { tracheostomy under general } \\
\text { intravenous anesthesia with } \\
\text { fiberoptic guidance at the } \\
\text { bedside }\end{array}$ & $\begin{array}{l}\text { Complications occurring during the } \\
\text { surgical procedure such as } \\
\text { bleeding, false passage, inability } \\
\text { to complete the procedure, } \\
\text { tracheal tears, pneumothorax, } \\
\text { and mortality }\end{array}$ \\
\hline $\begin{array}{l}\text { Johnson } \\
\text { et } \mathrm{al}^{21}\end{array}$ & $\begin{array}{l}\text { Adult subjects in an ICU with } \\
\text { indications for tracheostomy } \\
\text { such as ventilator dependence, } \\
\text { inability to protect the airway } \\
\text { due to decreased mental status, } \\
\text { facial trauma, and difficulty } \\
\text { weaning from a ventilator }\end{array}$ & $\begin{array}{l}\text { Blue Rhino tracheostomy } \\
\text { under general intravenous } \\
\text { anesthesia without } \\
\text { fiberoptic guidance at the } \\
\text { bedside }\end{array}$ & $\begin{array}{l}\text { Ciaglia multiple dilator } \\
\text { tracheostomy under general } \\
\text { intravenous anesthesia without } \\
\text { fiberoptic guidance }\end{array}$ & $\begin{array}{l}\text { Complications occurring during the } \\
\text { surgical procedure such as } \\
\text { bleeding, false passage, inability } \\
\text { to complete the procedure, } \\
\text { tracheal tears, pneumothorax, } \\
\text { and mortality }\end{array}$ \\
\hline $\begin{array}{l}\text { Byhahn } \\
{\text { et } \mathrm{al}^{22}}\end{array}$ & $\begin{array}{l}\text { Critically ill adult subjects } \\
\text { requiring elective tracheostomy } \\
\text { because of long-term } \\
\text { ventilation; excluding local } \\
\text { infection, endotracheal } \\
\text { intubation difficulty, distorted } \\
\text { anatomy, and unstable cervical } \\
\text { spine }\end{array}$ & $\begin{array}{l}\text { Blue Rhino tracheostomy } \\
\text { under general intravenous } \\
\text { anesthesia with fiberoptic } \\
\text { guidance at the bedside }\end{array}$ & $\begin{array}{l}\text { Ciaglia multiple dilator } \\
\text { tracheostomy under general } \\
\text { intravenous anesthesia with } \\
\text { fiberoptic guidance }\end{array}$ & $\begin{array}{l}\text { Complications such as bleeding, } \\
\text { ring fracture, posterior tracheal } \\
\text { wall perforation, pneumothorax, } \\
\text { and desaturation }\end{array}$ \\
\hline $\begin{array}{l}\text { Yurtseven } \\
\text { et } \mathrm{al}^{23}\end{array}$ & $\begin{array}{l}\text { Adult subjects requiring } \\
\text { endotracheal intubation for } \\
>10 \mathrm{~d} \text { due to acute respiratory } \\
\text { stress syndrome, infections, or } \\
\text { cerebrovascular events; } \\
\text { excluding prior tracheostomy, } \\
\text { hemorrhagic disorders, } \\
\text { anatomic abnormalities of the } \\
\text { trachea or cervical region, } \\
\text { infection, and need for an } \\
\text { emergency airway }\end{array}$ & $\begin{array}{l}\text { Griggs and PercuTwist } \\
\text { tracheostomy under general } \\
\text { intravenous anesthesia with } \\
\text { fiberoptic guidance at the } \\
\text { bedside }\end{array}$ & $\begin{array}{l}\text { Ciaglia multiple dilator } \\
\text { tracheostomy under general } \\
\text { intravenous anesthesia with } \\
\text { fiberoptic guidance }\end{array}$ & $\begin{array}{l}\text { Complications such as hypoxemia, } \\
\text { cardiovascular events, technical } \\
\text { difficulties, subcutaneous } \\
\text { emphysema, procedural } \\
\text { mortality, tracheal tears, tracheal } \\
\text { stenosis, false passage, tracheo- } \\
\text { oesophageal fistula, and bleeding }\end{array}$ \\
\hline
\end{tabular}

There were no deaths related to the procedure. In 2012, Kumar et al $^{13}$ compared the Ciaglia Blue Rhino versus Griggs dilating forceps methods in 30 subjects, with the Blue Rhino as the control group. Operators were anesthesiologist working in ICUs with previous experience. There were no data about the diseases of the subjects or indications for tracheostomy. Duration of intubation was not reported. The operating time was shorter in the Griggs group (11.7 vs $13.9 \mathrm{~min}$ ), but the difference was not statistically significant. Time to decannulation was not reported. Oxygen desaturation was higher in the Blue Rhino group (33\% vs 6.6\%); this was not statistically significant. There were more cases of airway loss in the Ciaglia group
( 2 vs 0 ). Karvandian et al $^{14}$ in 2012 compared the Ciaglia Blue Rhino versus Griggs dilating forceps methods in 100 subjects and considered the Blue Rhino as the control group. Operators were anesthesiologists with previous experience. Sixty-five subjects had pulmonary disorders, and 35 subjects had neurological disorders. Eighty-five subjects had an intubation duration of $>10 \mathrm{~d}$. The operating time was shorter than $5 \mathrm{~min}$ in the Griggs group (82\% in the Griggs group vs $24 \%$ in the Ciaglia group), which was statistically significant. Time to decannulation was not reported. Minimal bleeding was higher in the Blue Rhino group (92\% vs 70\%), and significant bleeding was similar between groups. 
Table 2. Events in Studies Comparing Different Techniques of Percutaneous Tracheostomy

\begin{tabular}{|c|c|c|c|c|c|c|c|c|c|c|c|c|}
\hline \multirow[b]{2}{*}{ Comparison } & \multirow[b]{2}{*}{$n$} & \multicolumn{11}{|c|}{ Outcome } \\
\hline & & $\begin{array}{l}\text { Tracheal } \\
\text { Ring } \\
\text { Fracture } \\
(n)\end{array}$ & $\begin{array}{c}\text { Posterior } \\
\text { Tracheal } \\
\text { Wall } \\
\text { Injury } \\
(n)\end{array}$ & $\begin{array}{l}\text { Pneumo- } \\
\text { thorax } \\
(n)\end{array}$ & $\begin{array}{c}\text { Oxygen } \\
\text { Desaturation } \\
<90 \%(n)\end{array}$ & $\begin{array}{l}\text { Major } \\
\text { Bleeding } \\
(n)\end{array}$ & $\begin{array}{c}\text { Loss of } \\
\text { Airway/ } \\
\text { Intubation } \\
(n)\end{array}$ & $\begin{array}{l}\text { Minor } \\
\text { Bleeding } \\
(n)\end{array}$ & $\begin{array}{l}\text { Difficulty } \\
\quad(n)\end{array}$ & $\begin{array}{l}\text { Puncture of } \\
\text { Endotracheal } \\
\text { Tube }(n)\end{array}$ & $\begin{array}{l}\text { Hypotension/ } \\
\text { Cardiac } \\
\text { Arrhythmia } \\
(n)\end{array}$ & $\begin{array}{l}\text { Overdilation } \\
\quad(n)\end{array}$ \\
\hline \multicolumn{13}{|l|}{ Byhahn et $\mathrm{al}^{22}$} \\
\hline $\begin{array}{l}\text { Ciaglia multiple } \\
\text { dilator }\end{array}$ & 25 & 2 & 2 & 1 & 1 & 0 & 3 & 3 & 5 & NR & NR & NR \\
\hline Ciaglia Blue Rhino & 25 & 9 & 0 & 0 & 2 & 0 & 1 & 4 & 2 & NR & NR & NR \\
\hline \multicolumn{13}{|l|}{ Johnson et $\mathrm{al}^{21}$} \\
\hline $\begin{array}{l}\text { Ciaglia multiple } \\
\text { dilator }\end{array}$ & 25 & NR & 0 & 0 & 0 & 0 & NR & NR & NR & NR & NR & NR \\
\hline Ciaglia Blue Rhino & 25 & NR & 0 & 0 & 1 & 0 & NR & NR & NR & NR & NR & NR \\
\hline \multicolumn{13}{|c|}{ Cianchi et $\mathrm{al}^{7}$} \\
\hline Ciaglia Blue Rhino & 35 & 3 & 0 & 0 & 0 & 0 & 0 & 12 & 2 & NR & NR & NR \\
\hline $\begin{array}{c}\text { Ciaglia Blue } \\
\text { Dolphin }\end{array}$ & 35 & 6 & 0 & 0 & 0 & 0 & 0 & 24 & 10 & NR & NR & NR \\
\hline \multicolumn{13}{|l|}{ Ambesh et al ${ }^{11}$} \\
\hline $\begin{array}{l}\text { Ciaglia Blue } \\
\text { Rhino }\end{array}$ & 30 & 9 & 2 & 1 & 0 & 0 & 0 & 1 & 2 & 2 & 2 & 0 \\
\hline Griggs & 30 & 0 & 0 & 0 & 0 & 0 & 0 & 5 & 9 & 3 & 0 & 7 \\
\hline \multicolumn{13}{|l|}{ Añon et $\mathrm{al}^{12}$} \\
\hline $\begin{array}{l}\text { Ciaglia Blue } \\
\text { Rhino }\end{array}$ & 27 & NR & 0 & 0 & 0 & 0 & 0 & 2 & 0 & NR & NR & NR \\
\hline Griggs & 26 & NR & 0 & 0 & 2 & 0 & 0 & 1 & 3 & NR & NR & NR \\
\hline \multicolumn{13}{|l|}{ Kumar et al ${ }^{13}$} \\
\hline $\begin{array}{l}\text { Ciaglia Blue } \\
\text { Rhino }\end{array}$ & 15 & 0 & 0 & 1 & 5 & 0 & 2 & NR & 2 & NR & 1 & 0 \\
\hline Griggs & 15 & 0 & 0 & 0 & 1 & 0 & 0 & NR & 1 & NR & 0 & 0 \\
\hline \multicolumn{13}{|l|}{ Karvandian et al ${ }^{14}$} \\
\hline $\begin{array}{l}\text { Ciaglia Blue } \\
\text { Rhino }\end{array}$ & 50 & 0 & 0 & 1 & 1 & 1 & 1 & 46 & 1 & NR & 6 & NR \\
\hline Griggs & 50 & 0 & 0 & 0 & 0 & 1 & 0 & 35 & 8 & NR & 5 & NR \\
\hline \multicolumn{13}{|l|}{ Birbicer et al ${ }^{24}$} \\
\hline $\begin{array}{l}\text { Ciaglia multiple } \\
\text { dilator }\end{array}$ & 50 & NR & 0 & 2 & 0 & 0 & NR & 2 & NR & NR & NR & NR \\
\hline PercuTwist & 50 & NR & 0 & 0 & 0 & 0 & NR & 2 & NR & NR & NR & NR \\
\hline \multicolumn{13}{|l|}{ Byhahn et a ${ }^{10}$} \\
\hline $\begin{array}{l}\text { Ciaglia Blue } \\
\text { Rhino }\end{array}$ & 35 & 1 & 0 & NR & 1 & 0 & 0 & 3 & 1 & NR & NR & NR \\
\hline PercuTwist & 35 & 1 & 2 & NR & 2 & 0 & 1 & 2 & 8 & NR & NR & NR \\
\hline \multicolumn{13}{|l|}{ Kaiser et al ${ }^{20}$} \\
\hline $\begin{array}{l}\text { Ciaglia multiple } \\
\text { dilator }\end{array}$ & 48 & NR & 2 & 1 & 6 & NR & 1 & 17 & 3 & NR & NR & NR \\
\hline Griggs & 52 & NR & 1 & 0 & 0 & NR & 0 & 6 & 0 & NR & NR & NR \\
\hline \multicolumn{13}{|l|}{ Nates et al ${ }^{18}$} \\
\hline $\begin{array}{l}\text { Ciaglia multiple } \\
\text { dilator }\end{array}$ & 52 & NR & NR & NR & NR & 1 & 0 & NR & 14 & NR & NR & NR \\
\hline Griggs & 48 & NR & NR & NR & NR & 7 & 2 & NR & 12 & NR & NR & NR \\
\hline \multicolumn{13}{|l|}{ Van Heurn et al ${ }^{16}$} \\
\hline $\begin{array}{l}\text { Ciaglia multiple } \\
\text { dilator }\end{array}$ & 63 & NR & NR & NR & NR & 2 & 0 & 2 & 2 & NR & NR & NR \\
\hline Griggs & 64 & NR & NR & NR & NR & 3 & 2 & 4 & 8 & NR & NR & NR \\
\hline \multicolumn{13}{|l|}{ Yurtseven et $\mathrm{al}^{23}$} \\
\hline $\begin{array}{l}\text { Ciaglia multiple } \\
\text { dilator }\end{array}$ & 44 & 1 & 1 & 0 & 0 & 0 & NR & 6 & 2 & NR & 0 & NR \\
\hline Griggs & 41 & 0 & 1 & 0 & 0 & 0 & NR & 4 & 1 & NR & 0 & NR \\
\hline PercuTwist & 45 & 0 & 0 & 0 & 0 & 0 & NR & 1 & 1 & NR & 0 & NR \\
\hline \multicolumn{13}{|l|}{ Cantais et $\mathrm{al}^{17}$} \\
\hline Griggs & 47 & NR & 0 & 0 & 0 & NR & 0 & 12 & 0 & NR & NR & NR \\
\hline Fantoni & 53 & NR & 2 & 0 & 3 & NR & 4 & 2 & 11 & NR & NR & NR \\
\hline \multicolumn{13}{|l|}{ Montcriol et $\mathrm{al}^{19}$} \\
\hline Griggs & 42 & NR & 0 & 0 & 0 & 1 & 1 & 2 & 0 & NR & NR & NR \\
\hline PercuTwist & 45 & NR & 0 & 0 & 1 & 0 & 2 & 1 & 4 & NR & NR & NR \\
\hline
\end{tabular}


Table 3. Methodological Quality of Studies Included

\begin{tabular}{|c|c|c|c|c|c|c|c|c|c|c|c|c|c|c|}
\hline & $\begin{array}{l}\text { Byhahn } \\
\text { et al }{ }^{10}\end{array}$ & $\begin{array}{l}\text { Van Heurn } \\
\text { et } \mathrm{al}^{16}\end{array}$ & $\begin{array}{l}\text { Ambesh } \\
\text { et } \mathrm{al}^{11}\end{array}$ & $\begin{array}{l}\text { Añon } \\
\text { et } \mathrm{al}^{12}\end{array}$ & $\begin{array}{l}\text { Cantais } \\
\text { et } \mathrm{al}^{17}\end{array}$ & $\begin{array}{l}\text { Nates } \\
\text { et al }{ }^{18}\end{array}$ & $\begin{array}{l}\text { Montcriol } \\
\text { et al }^{19}\end{array}$ & 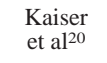 & 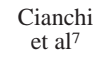 & $\begin{array}{l}\text { Johnson } \\
\text { et } \mathrm{al}^{21}\end{array}$ & $\begin{array}{l}\text { Byhahn } \\
\text { et } \mathrm{al}^{22}\end{array}$ & $\begin{array}{l}\text { Yurtseven } \\
\text { et } \mathrm{al}^{23}\end{array}$ & $\begin{array}{l}\text { Kumar } \\
\text { et al }{ }^{13}\end{array}$ & $\begin{array}{c}\text { Karvandian } \\
\text { et } \mathrm{al}^{14}\end{array}$ \\
\hline $\begin{array}{l}\text { Random } \\
\text { sequence } \\
\text { generation }\end{array}$ & Low risk & Low risk & Low risk & Low risk & Low risk & Unclear & Low risk & Low risk & Low risk & Low risk & Low risk & Low risk & Low risk & High risk \\
\hline $\begin{array}{l}\text { Allocation } \\
\text { concealment }\end{array}$ & Unclear & Unclear & Unclear & Unclear & Low risk & Unclear & Low risk & Low risk & Unclear & Low risk & Unclear & Low risk & Low risk & High risk \\
\hline $\begin{array}{l}\text { Blinding of } \\
\text { participants } \\
\text { and personnel }\end{array}$ & Low risk & Low risk & Low risk & Low risk & Low risk & Low risk & Low risk & Low risk & Low risk & Low risk & Unclear & Low risk & Low risk & Low risk \\
\hline $\begin{array}{l}\text { Blinding of } \\
\text { outcome } \\
\text { assessment }\end{array}$ & Low risk & Low risk & Low risk & Low risk & Low risk & Low risk & Low risk & Low risk & Low risk & Low risk & Low risk & Low risk & Low risk & Low risk \\
\hline $\begin{array}{l}\text { Incomplete } \\
\text { outcome } \\
\text { data }\end{array}$ & Low risk & Low risk & Low risk & Low risk & Low risk & Low risk & High risk & Low risk & Low risk & Low risk & Low risk & Low risk & Low risk & Low risk \\
\hline $\begin{array}{l}\text { Selective } \\
\text { reporting }\end{array}$ & Low risk & Low risk & Low risk & Low risk & Low risk & Low risk & Low risk & Low risk & Low risk & Low risk & Low risk & Low risk & Low risk & Low risk \\
\hline Other & Low risk & Low risk & Low risk & Low risk & Low risk & High risk & Low risk & Low risk & Low risk & Low risk & Low risk & Low risk & Low risk & Low risk \\
\hline
\end{tabular}

Risk differences were $-9.5 \%$ (95\% CI -15.7 to -3.3 ) for tracheal ring fracture, $-7.5 \%$ (95\% CI -16.1 to 1.2$)$ for minor bleeding, $13.2 \%$ (95\% CI 5.6-20.8) for difficulty, and $15.6 \%$ (95\% CI 3.9-27.2) for overdilation. There was no major bleeding or loss of airway/intubation. Comparisons of other outcomes were not statistically significant.

\section{Ciaglia Multiple Dilator Versus PercuTwist}

In 2008, Birbicer et al ${ }^{24}$ compared these 2 methods in 100 subjects. Operators were anesthesiologists, but there was no information about their experience, the subjects' conditions, or indications for tracheostomy. Duration of intubation was $\sim 7 \mathrm{~d}$. The operating time was shorter with the PercuTwist method (2.9 vs $4.1 \mathrm{~min}$ ). Data about time to cannulation were not provided. There were no statistically significant differences in outcome comparisons. There were no events of posterior tracheal wall injury, oxygen desaturation, or major bleeding.

\section{Ciaglia Blue Rhino Versus PercuTwist}

In 2002, Byhahn et $\mathrm{al}^{10}$ compared these 2 methods in 70 subjects, with the Blue Rhino as the control group. There were no data about operators and their experience, the subjects' conditions, indications for tracheostomy, or length of the procedure. Time to intubation was $\sim 9 \mathrm{~d}$. There were no deaths related to the procedure. The risk difference for difficulty was $-20 \%$ (95\% CI -35 to -5 ). There were no events of major bleeding, and comparisons of other outcomes were not statistically significant.

\section{Ciaglia Multiple Dilator Versus Griggs}

In 2000, Nates et al ${ }^{18}$ compared these 2 methods in 100 subjects, with the Ciaglia method considered as the control group. Operators were intensivists with previous experience. Results were stratified according to experience without differences in complications. Duration of intubation was $6.5 \mathrm{~d}$. Most subjects were trauma patients (46\%), and the main indication for tracheostomy was prolonged intubation (90\%). The length of the procedure was shorter in the Griggs group (6 vs $7 \mathrm{~min}$ ), but the difference was not statistically significant. Difficulty measured on a numerical scale showed a higher grade in the Griggs group (1.8 vs 1.3); this was statistically significant. Anesthesia complications, including hypotension, premature extubation, new atrial fibrillation, and desaturation, were equally distributed across both groups. There were no deaths related to the procedure. In 2001, Van Heurn et al ${ }^{16}$ compared the 2 methods in 127 subjects. The tracheostomies were done by or under the supervision of surgeons. There were no data about experience or the subjects' medical conditions. The main indication for tracheostomy was prolonged intubation (67\%). Duration of intubation was $\sim 14 \mathrm{~d}$, and time to decannulation was $\sim 21 \mathrm{~d}$. The length of the procedure was shorter for the Griggs method (5 vs $6 \mathrm{~min}$ ), and the difference was not statistically significant. There were no deaths related to the procedures. In 2006, Kaiser et $\mathrm{al}^{20}$ compared the 2 methods in 100 subjects. Operators were intensivists with previous experience. Most subjects had neurological conditions (63\%). There were no data about indications for tracheostomy. Duration of intubation was $\sim 8 \mathrm{~d}$. The length of the procedures was shorter for the Griggs method (4 vs $7 \mathrm{~min}$ ). There were no differences in $\mathrm{P}_{\mathrm{aO}_{2}}, \mathrm{P}_{\mathrm{CO}_{2}}, \mathrm{pH}$, arterial pressure, or heart rate measurements between groups. There were no deaths related to the procedures. There were no statistically significant differences in outcome comparisons. 


\section{Ciaglia Multiple Dilator Versus PercuTwist Versus Griggs}

In 2007, Yurtseven et $\mathrm{al}^{23}$ compared these 3 methods in 130 subjects, with the Ciaglia method as the control group. Operators were anesthesiologists with previous experience. Most subjects had pulmonary disorders (82\%). Time of intubation was $\sim 15 \mathrm{~d}$, and time to decannulation was $\sim 34 \mathrm{~d}$. The lengths of the procedures were shorter for the Percu Twist method (5.4 min) versus the Griggs $(6.2 \mathrm{~min})$ and Ciaglia $(9.9 \mathrm{~min})$ methods. No deaths related to the procedures were reported. There were no statistically significant differences in outcome comparisons. There were no events of pneumothorax, oxygen desaturation, major bleeding, or hypotension.

\section{Griggs Versus Fantoni}

In 2002, Cantais et al ${ }^{17}$ compared these 2 methods in 100 subjects. Operators were surgeons and anesthesiologists, but there was no information about their experience. Most subjects had neurological conditions (44\%), but there were no data about indications for tracheostomy or time to decannulation. Duration of intubation was $\sim 18 \mathrm{~d}$. The length of the procedure was shorter for the Griggs method (6.9 vs 12.9 min). There were no differences in $\mathrm{P}_{\mathrm{aO}_{2}}, \mathrm{P}_{\mathrm{CO}_{2}}$, and $\mathrm{pH}$ measurements between groups, but there was an increase in arterial pressure and heart rate in the translaryngeal method. No deaths related to the procedures were reported. The risk difference for difficulty was $-20.8 \%$ (95\% CI -317 to -9.8 ). Comparisons of other outcomes were not statistically significant.

\section{Griggs Versus PercuTwist}

In 2011, Montcriol et al ${ }^{19}$ compared these 2 methods in 87 subjects. Operators were intensivists with previous experience. Most subjects had neurological conditions (44\%). There were no data about indications for the procedure or time to decannulation. Duration of intubation was $\sim 10 \mathrm{~d}$. There were no differences in $\mathrm{P}_{\mathrm{aO}_{2}}, \mathrm{P}_{\mathrm{CO}_{2}}, \mathrm{pH}$, arterial pressure, heart rate, or intracranial pressure measurements between the groups. The length the procedure was shorter for the Griggs method ( 3 vs $5 \mathrm{~min}$ ). There were no deaths related to the procedures. There were no statistically significant differences in outcome comparisons. There were no events of posterior tracheal wall injury and pneumothorax.

A comparison was made between the Blue Rhino versus the other methods because the former is the most used device. ${ }^{10-14}$ The pooled risk differences were $0 \%(95 \% \mathrm{CI}$ -3.7 to $3.7, \mathrm{I}^{2} 0 \%$ ) for posterior tracheal wall injury, $-2.5 \%$ (95\% CI -6.6 to $1.7, \mathrm{I}^{2} 0 \%$ ) for pneumothorax, $-1.3 \%\left(95 \% \mathrm{CI}-5.7\right.$ to $\left.3.2, \mathrm{I}^{2} 38.5 \%\right)$ for oxygen de- saturation, $-6.3 \%$ (95\% CI -13.5 to $0.8, \mathrm{I}^{2} 74.2 \%$ ) for minor bleeding, $14.7 \%$ (95\% CI $8-21.5, \mathrm{I}^{2} 25.7 \%$ ) for difficulty, $0 \%\left(95 \% \mathrm{CI}-3.0\right.$ to $\left.3.0, \mathrm{I}^{2} 0 \%\right)$ for major bleeding, and $-16.5 \% 95 \%$ CI $\left(2.1\right.$ to $\left.-31.0, \mathrm{I}^{2} 0 \%\right)$ for total complications. It was not possible to make the same comparison for the Griggs method versus the other methods due to lack of outcome data.

\section{Discussion}

Tracheostomy has a long history in surgery. Excellent reviews about this subject show that the procedure was common and had good results even in ancient Egypt. ${ }^{1,25}$ The procedure remained unchanged for $>20$ centuries. In the middle of the 20th century, Shelden et al ${ }^{26}$ modified and described the first attempt of a less invasive tracheostomy, but it was not successful. Later in 1985, Pasquale Ciaglia, a thoracic surgeon in Utica, New York, first described 26 patients treated with a technique of percutaneous dilational tracheostomy based on the Seldinger principle, using a nephrostomy kit. ${ }^{2}$ In 1990, William M Griggs, an intensivist from Adelaide, Australia, described his method using guidewire dilating forceps, which were adapted Kelly clamps that allowed the passage of the wire to guide tracheal dilation. ${ }^{4}$ In 1995, Antonio Fantoni, from the anesthesia group at the San Carlo Borromeo Hospital in Milan, Italy, described the method of translaryngeal tracheostomy using the previously described procedure of Ciaglia, but in a retrograde fashion. ${ }^{8}$ In 2002, Giulio Frova, from the anesthesia department of the Civil Hospital in Brescia, Italy, described the method using a screw-like dilator. ${ }^{5}$ In 2005, Michael A Zgoda, ${ }^{6}$ from the pulmonary department at the University of Kentucky, described balloon dilation used with the Ciaglia Blue Rhino method.

All these techniques were designed with the specific objective of making a small incision with consequently less wound complications, simplifying the procedure so that it could be done at the bedside without an anesthesiologist or intubation while avoiding moving the patient to the operating room. However, some methods offer advantages over the others. For example, with the Griggs method, insertion is under direct vision and with only one dilation compared with the Ciaglia multiple dilator technique. The Fantoni method guarantees that the tube is intratracheal, and the Frova method tries to decrease the risk of cartilage fracture.

Although there is clear evidence of clinical equivalence between open and percutaneous tracheostomies, ${ }^{27-30}$ there is not enough information to conclude which of the percutaneous tracheostomy techniques is the best in terms of insertion success and complication rates.

In this review, 14 RCTs comparing different methods of percutaneous tracheostomy were found. The main features of each study are shown in Table 1. Most studies, except 


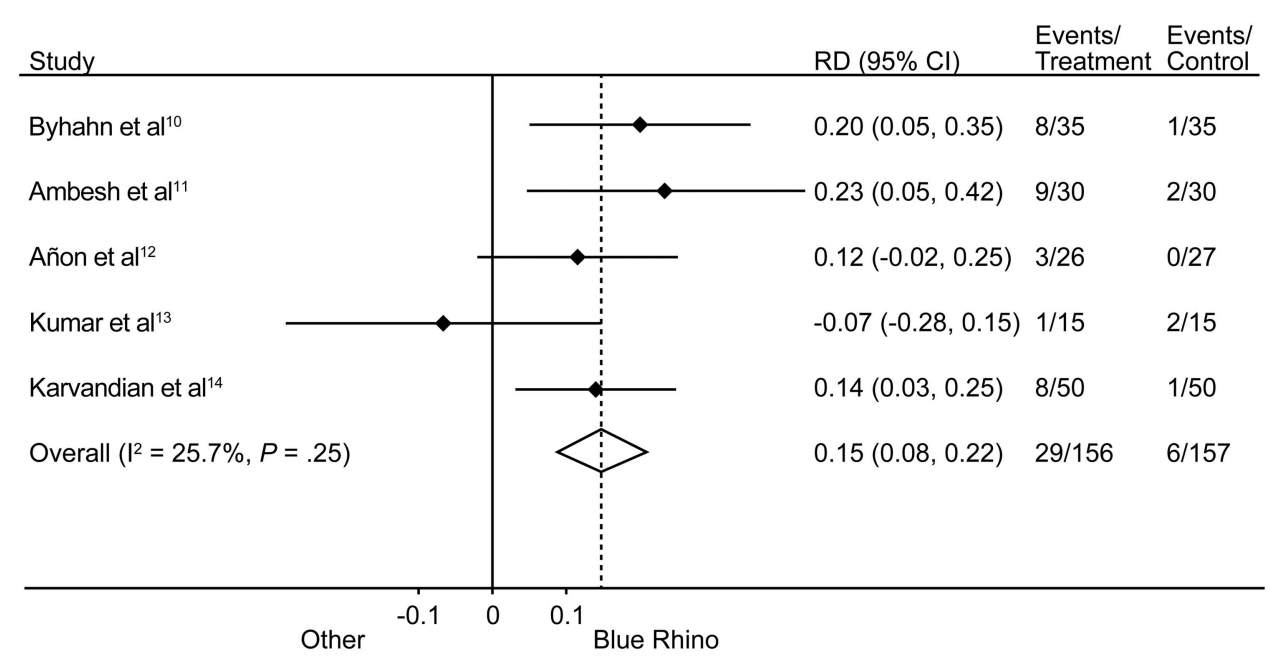

Fig. 2. Forrest plot for difficulty of the Blue Rhino method versus other methods. RD = risk difference.

that by Karvandian et al, ${ }^{14}$ are classified as low bias risk according to the Cochrane Collaboration criteria (see Table 2). Two studies compared the original Ciaglia technique with its modern version, the Ciaglia Blue Rhino method. The risk of tracheal ring fracture was the only statistically significant outcome that was higher in the Blue Rhino method. It was not possible to find a statistical difference in the other outcomes, but for most of the outcomes of these comparisons, only data from one study could be used. Only one study compared the Blue Rhino and Blue Dolphin methods, with more statistically significant events of minor bleeding and more subjective difficulty in the latter without differences in other outcomes. It was not possible to find information about the total number of complications. Comparison between the Blue Rhino and Griggs methods revealed a statistically significant difference in subjective tracheal ring fracture, minor bleeding, difficulty, and overdilation with the Griggs method, without differences in other outcomes. Comparisons between the original Ciaglia and PercuTwist methods and between the Ciaglia, Griggs, and PercuTwist methods did not indicate a statistically significant difference in any outcomes. Comparison between the Blue Rhino and PercuTwist showed that only difficulty was statistically different (higher for PercuTwist), but Birbicer et $\mathrm{al}^{24}$ did not report tracheal ring fractures, and Byhahn et $\mathrm{al}^{22} \mathrm{did}$ not report pneumothorax incidences.

In the comparison between the original Ciaglia and Griggs methods, no statistically significant difference were found in any outcome, with only a minor trend for major bleeding with the Griggs method. However, not all outcomes were reported by the trials, so many comparisons were made with data from only one or 2 trials. Comparison between the Griggs and Fantoni methods showed higher difficulty with the Fantoni method, without differences in other outcomes, whereas comparison between the Griggs and PercuTwist methods showed no differences in any outcomes.

As is evident from the above, comparisons between individual methods did not offer data to determine which method is superior because of the low number of trials, the incomplete number of events in some comparisons, and the small sample sizes in others. Therefore, a comparison was made between the Blue Rhino method and the other methods combined based on the availability and wide use of the Blue Rhino method. A lower subjective difficulty and more minor bleeding with the Blue Rhino method were found (Fig. 2), but with an important statistical heterogeneity as defined by the Higgins $\mathrm{I}^{2}$, which suggests that the pooled results are not conclusive. There was no higher major bleeding with the Blue Rhino method (Fig. 3 ). The other outcomes were not different, but had the same problems as with the individual analyses. A finding that was clear in all studies is a shorter time with the simple dilator method. However, time differences of a few minutes probably do not have a clinical impact on patients.

When new devices are designed and introduced in clinical practice, it is obligatory to assess their effectiveness and safety. Some devices are totally different from existing ones, and the way to assess effectiveness is through RCTs under a superiority hypothesis. ${ }^{31,32}$ However, most devices introduce only minor improvements compared with existing products; in such cases, the way to assess effectiveness and safety is in trials based on equivalence or non-inferiority hypotheses. ${ }^{31-33}$ This applies to percutaneous tracheostomy methods. After Ciaglia's design based on the Seldinger principle, the newer methods introduced only small changes designed to increase simplicity, speed, or safety of the previous methods. The equivalence trials are highly exigent due to the large sample size necessary to probe the hypothesis. In recent years, the appearance of the non-inferiority trial has offered a novel method that 


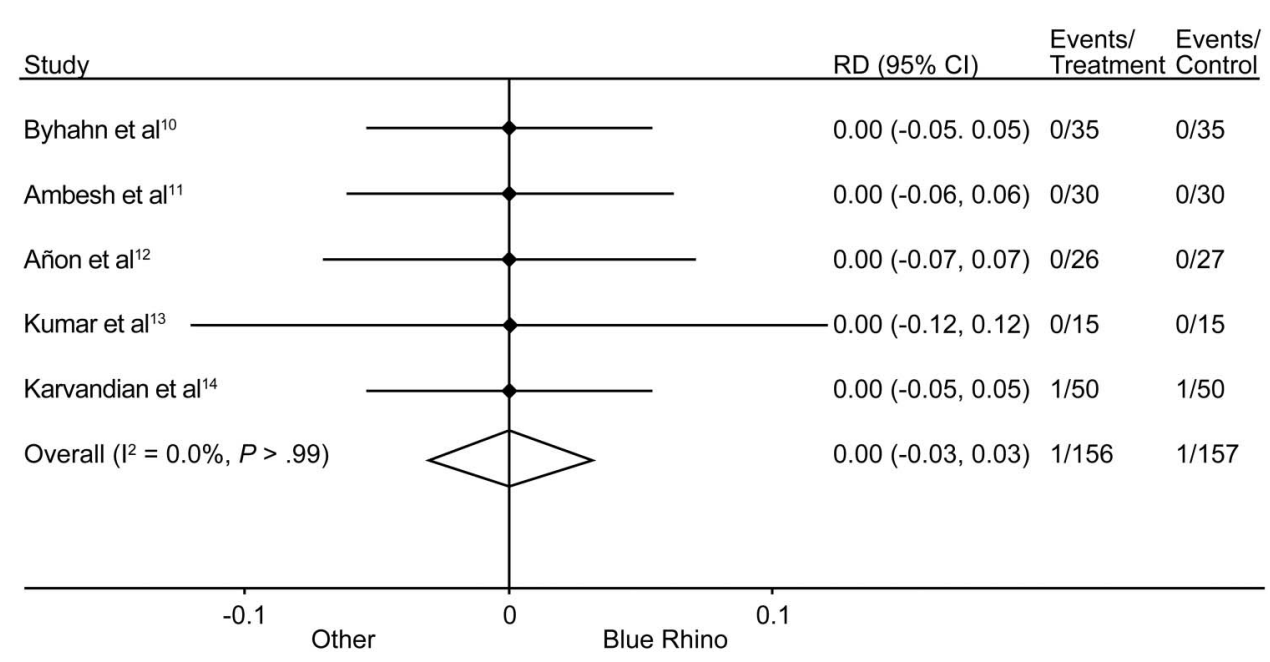

Fig. 3. Forrest plot for major bleeding of the Blue Rhino method versus other methods. RD = risk difference.

partially resolves the problem of huge sample sizes. If we want to test the equivalence of different percutaneous tracheostomy methods based on the low number of serious complications or rates of difficulty, $\sim 0-6 \%^{34}$ and a tolerable clinical difference of $2 \%$, it will be necessary to collect $>6,500$ patients. However, if a non-inferiority hypothesis is chosen, the sample size will probably decrease to 1,500 patients. This information helps one to understand the challenge of assessing the methods of percutaneous tracheostomy. Although 14 randomized trials were found, different methods were evaluated with different control groups and small sample sizes, making it difficult to support an equivalence hypothesis. Moreover, none of the studies included considered the non-inferiority hypothesis, so it is hard to determine that one method is not inferior to another. The methodological evaluation of the trials showed that although they complied with most of the Cochrane criteria to assess bias, the design and small sample size preclude reaching a clinically relevant conclusion. The problem of lack of power has been a constant in clinical research, and trials designed without a large enough sample size have even been considered unethical by some authors. ${ }^{35}$ In 2012, Cabrini et al ${ }^{9}$ tried to solve this problem with a meta-analysis. However, I differ from their approach. I excluded one of the trials they included, ${ }^{15}$ so data about events and number of total complications were different from this study. Although Cabrini et $\mathrm{al}^{9}$ found a high statistical heterogeneity, they accepted the pooled result, which is not recommended by meta-analysis experts. ${ }^{36-38}$

Most authors in the individual studies and in the only existing meta-analysis suggested that treatments are equivalent, but the type- 2 error precludes support for this conclusion, so it is not possible to determine whether one of the percutaneous method is better than another.
Another problem with the studies that were evaluated is the heterogeneity in defining outcomes. Complications were defined differently in the studies. For some authors, bleeding was major when requiring any form of intervention, but others defined it by quantity. Outcomes reported were also heterogeneous. Some reported all complications, but others reported only some, which decreases the number of eligible subjects to be included in a meta-analysis. The subjective aspect of surgical difficulty in introduction of a guidewire or dilation of the trachea was also a problem because the definition of these outcomes was difficult to standardize. Thus, it is probable that pooled results do not reflect reality about outcomes.

\section{Conclusions}

There are different methods of percutaneous tracheostomy, but it is impossible to determine which is better. I can only suggest that the Blue Rhino method is less difficult and probably has more minor bleeding events, but physicians also have more experience with this technique, so it is preferred over other methods. However, selection of each method must be made based on clinical criteria, experience, and availability. A larger RCT must be designed to compare the most common Blue Rhino method with the others based on previous calculation of sample size and a standard definition of important outcomes that allow this problem to be solved. It is also important to realize that percutaneous tracheostomy has been tested only in low-risk patients. Therefore, open tracheostomy is still the standard of care for most patients with adverse anatomical or ventilatory factors, even in ICUs with experience in percutaneous tracheostomy. 


\section{Percutaneous Tracheostomy Methods}

\section{REFERENCES}

1. Pratt LW, Ferlito A, Rinaldo A. Tracheotomy: historical review. Laryngoscope 2008;118(9):1597-1606.

2. Ciaglia P, Firsching R, Syniec C. Elective percutaneous dilatational tracheostomy. A new simple bedside procedure; preliminary report. Chest 1985;87(6):715-719.

3. Ciaglia P. Technique, complications, and improvements in percutaneous dilatational tracheostomy. Chest 1999;115(5):1229-1230.

4. Griggs WM, Worthley LI, Gilligan JE, Thomas PD, Myburg JA. A simple percutaneous tracheostomy technique. Surg Gynecol Obstet 1990;170(6):543-545.

5. Frova G, Quintel M. A new simple method for percutaneous tracheostomy: controlled rotating dilation. A preliminary report. Intensive Care Med 2002;28(3):299-303.

6. Zgoda MA, Berger R. Balloon-facilitated percutaneous dilational tracheostomy tube placement: preliminary report of a novel technique. Chest 2005;128(5):3688-3690.

7. Cianchi G, Zagli G, Bonizzoli M, Batacchi S, Cammelli R, Biondi S, et al. Comparison between single-step and balloon dilatational tracheostomy in intensive care unit: a single-centre, randomized controlled study. Br J Anaesth 2010;104(6):728-732.

8. Fantoni A, Ripamonti D. A non-derivative, non-surgical tracheostomy: the translaryngeal method. Intensive Care Med 1997;23(4): 386-392.

9. Cabrini L, Monti G, Landoni G, Biondi-Zoccai G, Boroli F, Mamo D, et al. Percutaneous tracheostomy, a systematic review. Acta Anaesthesiol Scand 2012;56(3):270-281.

10. Byhahn C, Westphal K, Meininger D, Gürke B, Kessler P, Lischke V. Single-dilator percutaneous tracheostomy: a comparison of PercuTwist and Ciaglia Blue Rhino techniques. Intensive Care Med 2002;28(9):1262-1266.

11. Ambesh SP, Pandey CK, Srivastava S, Agarwal A, Singh DK. Percutaneous tracheostomy with single dilatation technique: a prospective, randomized comparison of Ciaglia Blue Rhino versus Griggs' guidewire dilating forceps. Anesth Analg 2002;95(6):1739-1745.

12. Añon JM, Escuela MP, Gómez V, Moreno A, López J, Díaz R, et al. Percutaneous tracheostomy: Ciaglia Blue Rhino versus Griggs' guide wire dilating forceps. A prospective randomized trial. Acta Anaesthesiol Scand 2004;48(4):451-456.

13. Kumar M, Trikha A, Chandralekha. Percutaneous dilatational tracheostomy: Griggs guide wire dilating forceps technique versus ULTRA-perc single-stage dilator-a prospective randomized study. Indian J Crit Care Med 2012;16(2):87-92.

14. Karvandian K, Yousefian M, Khan ZH, Baigmohammadi T, Shabani S. Comparative clinical trial between Ciaglia and Griggs techniques during tracheostomy performed in patients admitted to intensive care unit. Acta Med Iran 2012;50(8):525-529.

15. Stocchetti N, Parma A, Lamperti M, Songa V, Tognini L. Neurophysiological consequences of three tracheostomy techniques: a randomized study in neurosurgical patients. J Neurosurg Anesthesiol 2000;12(4):307-313.

16. Van Heurn LW, Mastboom WB, Scheeren CI, Brink PR, Ramsay G. Comparative clinical trial of progressive dilatational and forceps dilatational tracheostomy. Intensive Care Med 2001;27(1):292-295

17. Cantais E, Kaiser E, Le-Goff Y, Palmier B. Percutaneous tracheostomy: prospective comparison of the translaryngeal technique versus the forceps-dilational technique in 100 critically ill adults. Crit Care Med 2002;30(4):815-819.
18. Nates JL, Cooper DJ, Myles PS, Scheinkestel CD, Tuxen DV. Percutaneous tracheostomy in critically ill patients: a prospective, randomized comparison of two techniques. Crit Care Med 2000;28(11): 3734-3739.

19. Montcriol A, Bordes J, Asencio Y, Prunet B, Lacroix G, Meaudre E. Bedside percutaneous tracheostomy: a prospective randomised comparison of PercuTwist versus Griggs' forceps dilational tracheostomy. Anaesth Intensive Care 2011;39(2):209-216.

20. Kaiser E, Cantais E, Goutorbe P, Salinier L, Palmier B. Prospective randomized comparison of progressive dilational vs forceps dilational percutaneous tracheostomy. Anaesth Intensive Care 2006; 34(1):51-54.

21. Johnson JL, Cheatham ML, Sagraves SG, Block EF, Nelson LD. Percutaneous dilational tracheostomy: a comparison of single- versus multiple-dilator techniques. Crit Care Med 2001;29(6):1251-1254.

22. Byhahn C, Wilke HJ, Halbig S, Lischke V, Westphal K. Percutaneous tracheostomy: Ciaglia Blue Rhino versus the basic Ciaglia technique of percutaneous dilational tracheostomy. Anesth Analg 2000; 91(4):882-886.

23. Yurtseven N, Aydemir B, Karaca P, Aksoy T, Komurcu G, Kurt M, et al. PercuTwist: a new alternative to Griggs and Ciaglia's techniques. Eur J Anaesthesiol 2007;24(6):492-497.

24. Birbicer H, Doruk N, Yapici D, Atici S, Altunkan AA, Epozdemir S, Oral U. Percutaneous tracheostomy: a comparison of PercuTwist and multi-dilatators techniques. Ann Card Anaesth 2008;11(2):131.

25. Ferlito A, Rinaldo A, Shaha AR, Bradley PJ. Percutaneous tracheotomy. Acta Otolaryngol 2003;123(9):1008-1012.

26. Shelden CH, Pudenz RH, Freshwater DB, Crue BL. A new method for tracheotomy. J Neurosurg 1955;12(4):428-431.

27. Cheng E, Fee WE Jr. Dilatational versus standard tracheostomy: a meta-analysis. Ann Otol Rhinol Laryngol 2000;109(9):803-807.

28. Delaney A, Bagshaw SM, Nalos M. Percutaneous dilatational tracheostomy versus surgical tracheostomy in critically ill patients: a systematic review and meta-analysis. Crit Care 2006;10(2):R55.

29. Freeman BD, Isabella K, Lin N, Buchman TG. A meta-analysis of prospective trials comparing percutaneous and surgical tracheostomy in critically ill patients. Chest 2000;118(5):1412-1418.

30. Higgins KM, Punthakee X. Meta-analysis comparison of open versus percutaneous tracheostomy. Laryngoscope 2007;117(3):447-454.

31. Goeman JJ, Solari A, Stijnen T. Three-sided hypothesis testing: simultaneous testing of superiority, equivalence and inferiority. Stat Med 2010;29(20):2117-2125.

32. Lesaffre E. Superiority, equivalence, and non-inferiority trials. Bull NYU Hosp Jt Dis 2008;66(2):150-154.

33. Cipriani A, Girlanda F, Barbui C. Superiority, equivalence or noninferiority? Epidemiol Psichiatr Soc 2009;18(4):311-313.

34. Kearney PA, Griffen MM, Ochoa JB, Boulanger BR, Tseui BJ, Mentzer RM Jr. A single-center 8-year experience with percutaneous dilational tracheostomy. Ann Surg 2000;231(5):701-709.

35. Altman DG. Statistics and ethics in medical research: III how large a sample? Br Med J 1980;281(6251):1336-1338.

36. Ioannidis JP. Interpretation of tests of heterogeneity and bias in meta-analysis. J Eval Clin Pract 2008;14(5):951-957.

37. Keefe SM, Strom BL. On the importance of heterogeneity in metaanalysis. Clin Trials 2009;6(5):443-444.

38. Song F, Sheldon TA, Sutton AJ, Abrams KR, Jones DR. Methods for exploring heterogeneity in meta-analysis. Eval Health Prof 2001; 24(2):126-151. 